OPEN ACCESS

Edited by:

Lucienne Chatenoud, Université Paris Descartes, France

Reviewed by: Fan Pan,

Chinese Academy of Sciences (CAS), China

Lianjun Zhang,

Suzhou Institute of Systems Medicine

(ISM), China

*Correspondence:

Christian Jorgensen christian.jorgensen@inserm.fr

Maroun Khoury

mkhoury@uandes.cl

Specialty section:

This article was submitted to Immunological Tolerance and Regulation,

a section of the journal

Frontiers in Immunology

Received: 15 September 2020 Accepted: 20 January 2021

Published: 08 March 2021

Citation: Jorgensen C and Khoury M (2021) Musculoskeletal Progenitor/Stromal Cell-Derived Mitochondria Modulate Cell Differentiation and

Therapeutical Function.

Front. Immunol. 12:606781. doi: 10.3389/fimmu.2021.606781

\section{Musculoskeletal Progenitor/Stromal Cell-Derived Mitochondria Modulate Cell Differentiation and Therapeutical Function}

\author{
Christian Jorgensen ${ }^{1,2,3 *}$ and Maroun Khoury ${ }^{4,5,6 *}$ \\ 1 Inserm, U1183, Montpellier, France, ${ }^{2}$ Université MONTPELLIER 1, UFR de Médecine, Montpellier, France, ${ }^{3}$ Service \\ d'immuno-Rhumatologie, Hôpital Lapeyronie, Montpellier, France, ${ }^{4}$ Laboratory of Nano-Regenerative Medicine, Centro de \\ Investigación e Innovación Biomédica (CIIB), Faculty of Medicine, Universidad de los Andes, Santiago, Chile, ${ }^{5}$ Cells for Cells, \\ Santiago, Chile, ${ }^{6}$ Consorcio Regenero, Chilean Consortium for Regenerative Medicine, Santiago, Chile
}

Musculoskeletal stromal cells' (MSCs') metabolism impacts cell differentiation as well as immune function. During osteogenic and adipogenic differentiation, BM-MSCs show a preference for glycolysis during proliferation but shift to an oxidative phosphorylation (OxPhos)-dependent metabolism. The MSC immunoregulatory fate is achieved with cell polarization, and the result is sustained production of immunoregulatory molecules (including PGE2, HGF, IL1RA, IL6, IL8, IDO activity) in response to inflammatory stimuli. MSCs adapt their energetic metabolism when acquiring immunomodulatory property and shift to aerobic glycolysis. This can be achieved via hypoxia, pretreatment with small molecule-metabolic mediators such as oligomycin, or AKT/mTOR pathway modulation. The immunoregulatory effect of MSC on macrophages polarization and Th17 switch is related to the glycolytic status of the MSC. Indeed, MSCs pretreated with oligomycin decreased the M1/M2 ratio, inhibited T-CD4 proliferation, and prevented Th17 switch. Mitochondrial activity also impacts MSC metabolism. In the bone marrow, MSCs are present in a quiescent, low proliferation, but they keep their multi-progenitor function. In this stage, they appear to be glycolytic with active mitochondria (MT) status. During MSC expansion, we observed a metabolic shift toward OXPhos, coupled with an increased MT activity. An increased production of ROS and dysfunctional mitochondria is associated with the metabolic shift to glycolysis. In contrast, when MSC underwent chondro or osteoblast differentiation, they showed a decreased glycolysis and inhibition of the pentose phosphate pathway (PPP). In parallel the mitochondrial enzymatic activities increased associated with oxidative phosphorylation enhancement. MSCs respond to damaged or inflamed tissue through the transfer of MT to injured and immune cells, conveying a type of signaling that contributes to the restoration of cell homeostasis and immune function. The delivery of MT into injured cells increased ATP levels which in turn maintained cellular bioenergetics and recovered cell functions. MSC-derived MT may be transferred via tunneling nanotubes to undifferentiated cardiomyocytes and leading to their maturation. In this review, we will decipher the pathways and the mechanisms 
responsible for mitochondria transfer and activity. The eventual reversal of the metabolic and pro-inflammatory profile induced by the MT transfer will open new avenues for the control of inflammatory diseases.

Keywords: musculoskeletal progenitor/stromal cells, immunosuppression, mitochondria, stem cell, immunometabolism

\section{INTRODUCTION}

Musculoskeletal pogenitor/stromal cells (MSCs) (also referred to as mesenchymal stem cells) have been proposed as a cell therapy for mesoderm-derived tissue regeneration and immune modulation. MSCs are the progenitors of mesoderm lineages including bone, cartilage, muscle, fat, tendon, and synovium. MSCs can be isolated from the bone marrow (BM) (1), adipose tissue (Ad) (2), umbilical cord blood (UCB), placenta, Wharton's jelly (3), dental pulp (4), or from induced pluripotent stem (IPS) cell-derived MSCs (5). Due to easy access and high productivity, autologous and allogeneic MSCs are currently most often obtained from bone marrow or adipose tissue, while allogeneic MSCs are also obtained from UCB or Wharton's jelly (3). Significant functional differences between MSC sources have been reported. The best strategy for MSC-based therapy has therefore to be determined according to their distinct characteristics associated with their tissue origin for a particular therapeutic application.

Variation in MSC sources, passage number during ex vivo culture, and age-related fatigue of MSC function and change of cell metabolism during expansion may result in variability in MSC function (6). This may induce heterogeneity in clinical trial results. Understanding of regulation of MSC metabolism is critical as that the manipulation of cell metabolism allows enhanced therapeutic uses of these cells (e.g., cell retention, cell survival, immunoregulation, differentiation) in cell-based medicine and tissue engineering. Here, we review the present knowledge on the metabolic pathways involved in the function of MSC and the perspectives for their optimal therapeutic applications.

\section{ENERGETIC METABOLISM IS CRITICAL IN MSC DIFFERENTIATION AND FUNCTION}

MSC metabolism impacts cell differentiation as well as immune function. BM-MSCs show a preference for glycolysis during proliferation. During proliferation, the human MSCs (hMSCs) primarily generate ATP through glycolysis (7) but shift to oxidative phosphorylation (OxPhos)-dependent metabolism during osteogenic and adipogenic differentiation (OD, $A D)$ (8), as depicted in Figure 1. In contrast, adipose derived ASC increased both glycolysis and mitochondrial metabolism associated with OxPhos and fatty acid b-oxidation (10). When Ad-hMSCs underwent adipogenesis, they showed a decreased capacity for the pentose phosphate pathway (PPP) and glycolysis, while mitochondrial enzyme activities increased in parallel to oxidative phosphorylation and b-oxidation (Figure 1).

To induce immune stimulation and polarization in hMSCs, activation through proinflammatory cytokine including $\mathrm{TNFa}$ and/or IFN-g is necessary. In response to this inflammatory stimuli, MSCs demonstrate immunoregulatory function associated with cell polarization and enhanced production of immunoregulatory factors (including PGE2, HGF, IL1RA, IL6, IL8, IDO activity) $(11,12)$. Interestingly, this MSC polarization requires a metabolic shift toward aerobic glycolysis.

Moreover, hypoxia enhanced MSC immunomodulatory function through a change in cell metabolism. Similar change in cell metabolism is obtained with pretreatment with small molecule-metabolic mediators such as oligomycin or AKT/ mTOR pathway modulation. The immunoregulatory effect of MSC on macrophage polarization and Th17switch is related to the glycolytic status of the MSC. MSCs pretreated with oligomycin decreased the M1/M2 ratio, inhibited T-CD4 proliferation, and prevented Th17 switch (13).

After cytokine stimulation, hMSC metabolism switches their metabolic pathways toward glycolysis (reducing TCA cycle metabolism), and this seems to be required for a sustained immunosuppressive effect (14). PGE2 and IDO activity increased in response to augmented aerobic glycolysis. Consequently, the hMSC activation was associated with increased expression of glucose transporter 1 and hexokinase isoform 2 (key enzymes in glycolysis), and reduced electron transport and OxPhos. Furthermore, activated MSCs exhibited a glycolytic phenotype and inhibited activity of the mitochondrial electron transport chain (complexes I or III), blocking OxPhos and reducing mitochondria-related reactive oxygen species (mROS) (14). The Akt/mTOR signaling pathway activation was shown to be key in the induction of metabolic switch (15). Through induction of mTOR activity, IFN increases the glycolytic capacity of the MSC. This energy metabolic modification enhances the MSC ability to inhibit T cell proliferation. As a result, the IFN-g induced expression of growth factors, including hepatocyte growth factor (HGF), that is involved in immune and antifibrotic functions of hMSCs (16).

\section{MSC EXPANSION AND ALTERATION IN THE METABOLISM}

To achieve sufficient numbers of MSCs for clinical transfer, cell expansion is a necessary step. This cell expansion is performed either in flask with a tight control of oxygen and nutriment and $\mathrm{CO}_{2}$ level or in bioreactors. In the bone marrow, hMSCs are present in a quiescent state with low cell proliferation maintained overtime. 


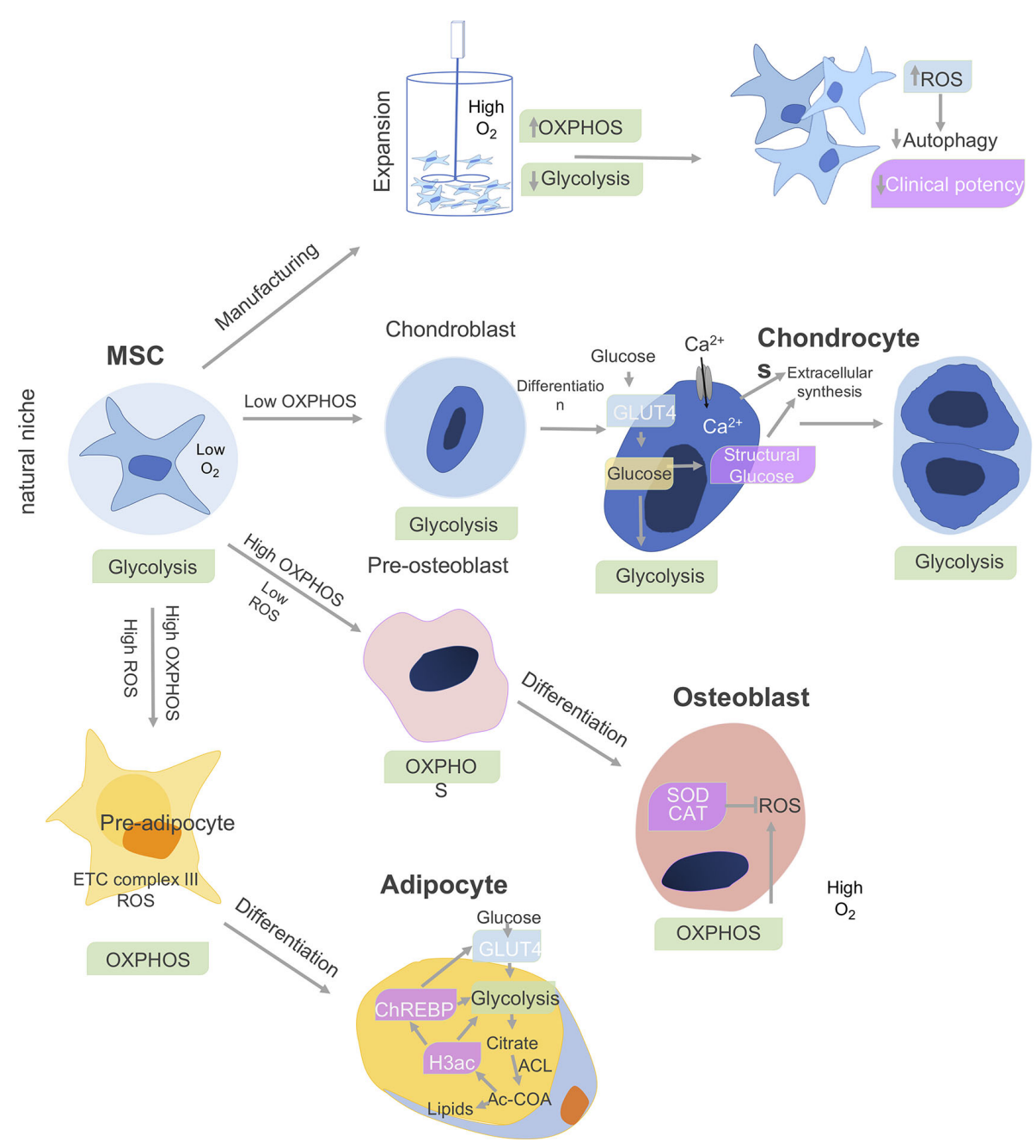

FIGURE 1 | Cell metabolism and MSC differentiation. The metabolism of MSCs changes depending on the niche and differentiation stages. The bone marrow environment is a hypoxic niche where MSCs remain quiescent and use glycolysis. When isolated and expanded ex vivo in a nutrient-rich artificial culture environment, proliferation is promoted, which derives a significant proportion of ATP from oxidative phosphorylation (OXPHOS) in addition to glycolysis. The accumulation of cytotoxic metabolic byproducts, including reactive oxygen species (ROS), reduces basal autophagy and mitophagy rates while increasing the fraction of senescent cells leading to reduced clinical potency. Under chondro-inductive differentiation conditions, glycolysis is further upregulated during chondrogenesis in chondroblasts and which gives rise to chondrocytes and cartilage. In osteoblasts, which give rise to bone, both $\mathrm{O}_{2}$ consumption and oxidative phosphorylation (OXPHOS) are upregulated, while superoxide dismutase (SOD) and catalase (CAT) negatively regulate ROS secretion. Finally, adipocyte differentiation is associated with upregulation of OxPhos metabolism, release reactive oxygen species (ROS), and electron transport chain (ETC) complex III. Adipocytes upregulate glycolysis and ATP citrate lyase (ACL), which leads to increased cytosolic acetyl-CoA (Ac-CoA) synthesis. In parallel, increase in histone H3 acetylation (H3ac) is associated with lipid synthesis. H3ac activates the carbohydrate-responsive element-binding protein (ChREBP) transcription factor to promote glycolysis through GLUT4-mediated glucose uptake (9).

In this undifferentiated fate, MSCs are in glycolytic metabolism with small mitochondria maintained by active autophagy and mitophagy (17). Inducing hMSC proliferation in a bioreactor leads to an enhanced significant ATP production derived from oxidative phosphorylation (OXPHOS) to support the proliferation (18). This results in accumulation of reactive oxygen species (ROS), reduction of the basal autophagy rate and dysfunctional mitochondria, and increase of senescent cells $(19,20)$. As the MSC function is associated with glycolytic state, bioreactor expansion leads to emergence of OXPHOS metabolic profile MSC with reduction if differentiation function (21) is associated with the accumulation of reactive oxygen species (ROS) (9). This leads to cell senescence and reduction in autophagy and mitophagy rates $(21)$

\section{REGULATION OF MSC METABOLISM TO IMPROVE CELL FUNCTION}

Hypoxia enhances hMSC immunoregulatory properties. In a GVHD preclinical study, hypoxic pretreatment of hMSC enhanced the secretion of IL-10 and Fas ligand and improved animal survival and weight loss (22). Overexpression of HIF1-a mimics hypoxia and is shown to enhance hMSC immunomodulatory properties (13). 
Preconditioning of the cells with $\mathrm{N}$-acetylcysteine (NAC), which stabilizes HIF-1, was shown to improve MSC anti-inflammation cell retention (23), and osteogenesis (24).

Pre-activation of MSCs with IFN has been widely reported to enhance MSC immunomodulatory properties as well as an inducer of the metabolic switch in preclinical models. For example, the infusion of IFN-g pretreated MSCs in an immunodeficient mouse model significantly reduced the symptoms of GVHD and improved survival (25).

Recently, we have shown that $\operatorname{PPAR} \beta / \delta$ is critical for the immunoregulatory functions of MSC. The upregulation of this transcription factor enhanced the expression of genes associated with fatty acid transport and $\beta$-oxidation (26). PPAR $\beta / \delta$ increases MSC glycolytic activity required for their immune impact on Th1 and Th17 cells (26). Moreover, $\operatorname{PPAR} \beta / \delta$ inhibited mitochondrial ATP production and promoted their metabolic switch towards aerobic glycolysis leading to enhancement of their immunosuppressive functions. Preconditioning MSC with $\operatorname{PPAR} \beta / \delta$ inhibitors improved MSC function through metabolic reprogramming and enhanced MSC immunoregulatory properties in arthritis models (26).

On the other hand, Notch signaling is a conserved pathway that regulates cell-fate determination during development and maintains adult tissue homeostasis. The activation of Notch by either Jagged1 or the Notch2 intracellular domain suppresses glucose metabolism in mesenchymal progenitors and inhibits their osteoblastic differentiation potential. AMP-activated protein kinase (AMPK) plays a critical role as regulator of cellular metabolic homeostasis. Notch downregulated AMPK activity and inhibited glycolysis in hMSC (27). Notch reduces the expression of mitochondrial complex I genes, resulting in a decrease in mitochondrial respiration, superoxide production. Thus Notch pathway prevented osteoblastogenesis from bone marrow mesenchymal progenitors, demonstrating that AMPK is critical for MSC differentiation potential (27).

The mechanistic Target of Rapamycin (mTOR) coordinates eukaryotic cell growth and metabolism with environmental inputs including nutrients and growth factors.

mTORC1 facilitates growth by promoting a shift in glucose metabolism from oxidative phosphorylation to glycolysis, which likely facilitates the incorporation of nutrients into new biomass (15). Rapamycin is an mTOR inhibitor used to precondition MSCs to upregulate their immunosuppressive functions. Rapamycin inhibited cyclooxygenase (COX)-2 and PGE2 expression. Rapamycin preconditioning of MSCs strongly inhibited Th17 cell expansion through the upregulation of IL-10, IDO, and TGF- $\beta$ (28). This confirmed that mTOR inhibition enhanced the therapeutic effect of MSCs in autoimmune diseases.

\section{MITOCHONDRIA TRANSFER, A NEW MSC INTERCELLULAR MODULATORY PATHWAY}

The requirement of cell-contact for MSCs to display immunoregulatory abilities is convergent with a set of recent observations that indicates that these cells can also achieve a range of effects by means of the transfer of their own mitochondria (MT) (29), to target renal, myocardial, or lung alveolar cells to restore target tissue homeostasis $(30,31)$. Thus, MSCs can also transfer this organelle to immunocompetent cells, conveying a type of signaling that contributes to the restoration of immune homeostasis (32). It is of considerable interest to elucidate this additional regulatory mechanism, potentially underlying immune function of MSCs.

The clinical significance of this phenomenon was first assessed in a model of lipopolysaccharide (LPS)-induced lung injury in which the intra-tracheal administration of MSCs to LPS treated mice was associated with the transfer of MT (MitoT) to the alveolar epithelium. MSCs triggered an increase in the concentration of ATP, metabolic activity and also an improvement in lung damage while reducing mortality in the diseased animals. The effects were dependent on the MSC expression of connexin 43 (CNX43) and the generation of nanotubes bridging alveolar cells (33). Subsequently, in an allergen mediated model of bronchial inflammation, MitoT from MSCs was shown to be controlled by Miro1, a MT Rho-GTPase (34). Again, MSC treatment reversed airway inflammation, hyper responsiveness and tissue damage. Exogenously administered MSCs were found to respond to tissular oxidative stress, rescuing epithelial cells by a process of MitoT that occurred via microtubules forming tunneling nanotubes (TNT) between cells. A set of elegant experiments showed these structures were the conduit responsible for the transportation of organelles by bridging MSCs with their target cells when submitted to stress (34). In another model of MSC co-cultured with vascular endothelial cells (VECs), the latter were able to induce proliferation of MSCs, a phenomenon partially suppressed with an inhibitor of TNT or by using VECs with defective MT (35). The relevance of MitoT from MSCs was also demonstrated in a model of ethidium bromide induced damage of MT in a cancer cell line. Treated cancer cells had reduced metabolic and proliferative capacity under standard conditions, an effect that was reversed by co-culture with MSCs. The rescue was linked to a restoration of the synthesis of nucleotides, found to be impaired by lack of functional MT in the cancer cell lines (36). More recently the transfer of MT from MSCs to macrophages has also been described. Jackson et al. observed that MT transferred from MSCs were able to increase the phagocytic capabilities of macrophages, increasing basal respiration and ATP turnover in vitro and in vivo in a murine model of acute respiratory distress syndrome (ARDS) (37). The mechanism by which MSCs transferred MT in this case was independent of CNX43 but related to the establishment of TNT between both cell types. Interestingly, the artificial transfer of MT from MSCs into macrophages conveys the same metabolic and phagocytic improvement, evidencing a role for MitoT in macrophage function (REF) (37). To date, it is well established that MSCs suppress $\mathrm{T}$ cell function (38). Given the relevance of the Th1/Th17 and Treg balance in the outcome of immune mediated diseases and the participation of MSCs in controlling this balance by means of cell-to-cell contact, MT transfer from MSCs to CD4+ T cell 
subpopulations plays a role in the regulation of $\mathrm{T}$ cell function, contributing to the dampening of inflammation that is associated with MSC therapy in graft versus host disease (GVHD) and other immune mediated conditions (39). Studies from our group showed immunosuppressive effects of MitoT from MSCs to lymphocytes, promoting Treg differentiation and inhibiting th 17 (40). In an experimental xeno-GVHD model, we showed that MT isolated from MSCs and transferred to human PBMCs before their infusion alleviates inflammatory responses leading to significant improvement in the survival and reduction in tissue damage and organ $\mathrm{T} \mathrm{CD} 4+, \mathrm{CD}^{+}$, and IFN $-\gamma^{+}$expressing cell infiltration (41). These results represent strong evidence in favor of the hypothesis that the transfer of MSC-derived MT to immune active cells could play a role in the control of immune function mediated by MSCs.

\section{MSC TRANSFER THEIR MT MAINLY THROUGH TUNNELING NANOTUBES}

Even though mitochondrial exchange has been well reported in the literature, the underlying mechanisms and signaling pathways associated with MT transfer remain to be elucidated. Upon injury, a distress signal is released by damaged cells that can be picked up by surrounding MSCs through their surface "sensors", triggering an immediate mobilization and a migration shift toward the injury site. The MSC that migrated to the injured tissue will release growth factors as well as IDO activity in response to the microenvironment stimulus. The role of MSCs in tissue homeostasis is also associated with organelle exchange to damaged recipient cells (42). The formation of channels and membrane-like structures that allow the transfer of MT between MSCs and the recipient cells has been visualized and described as tunneling nanotubes (TNTs), vesicles, gap junctions, and fusion $(37,43)$. As described by Rustom et al. TNTs are long-distance tubular structures or projections that have a diameter between 50 and $150 \mathrm{~nm}$ and are able to connect different cells together (44). TNTs do not attach to the substrate of the cell nor are tethered to the extracellular matrix (Figure 2). Release and donation are not limited to MT as other organelles and cellular structures can also transit including extracellular and intracellular vesicles, Golgi, and lysosomal vesicles in a highly well-orchestrated process. The formation of TNTs and organelle motility inside these structures are well-regulated process by proteins known as TNFAIP2 and Miro1 along with accessory proteins, such as Miro2, TRAK1, $\mathrm{KHC}$, and Myo19. The inhibition of Mirol in MSC does not prevent the formation of TNT but retards mitochondrial movement, showing that mitochondrial donation is a well-

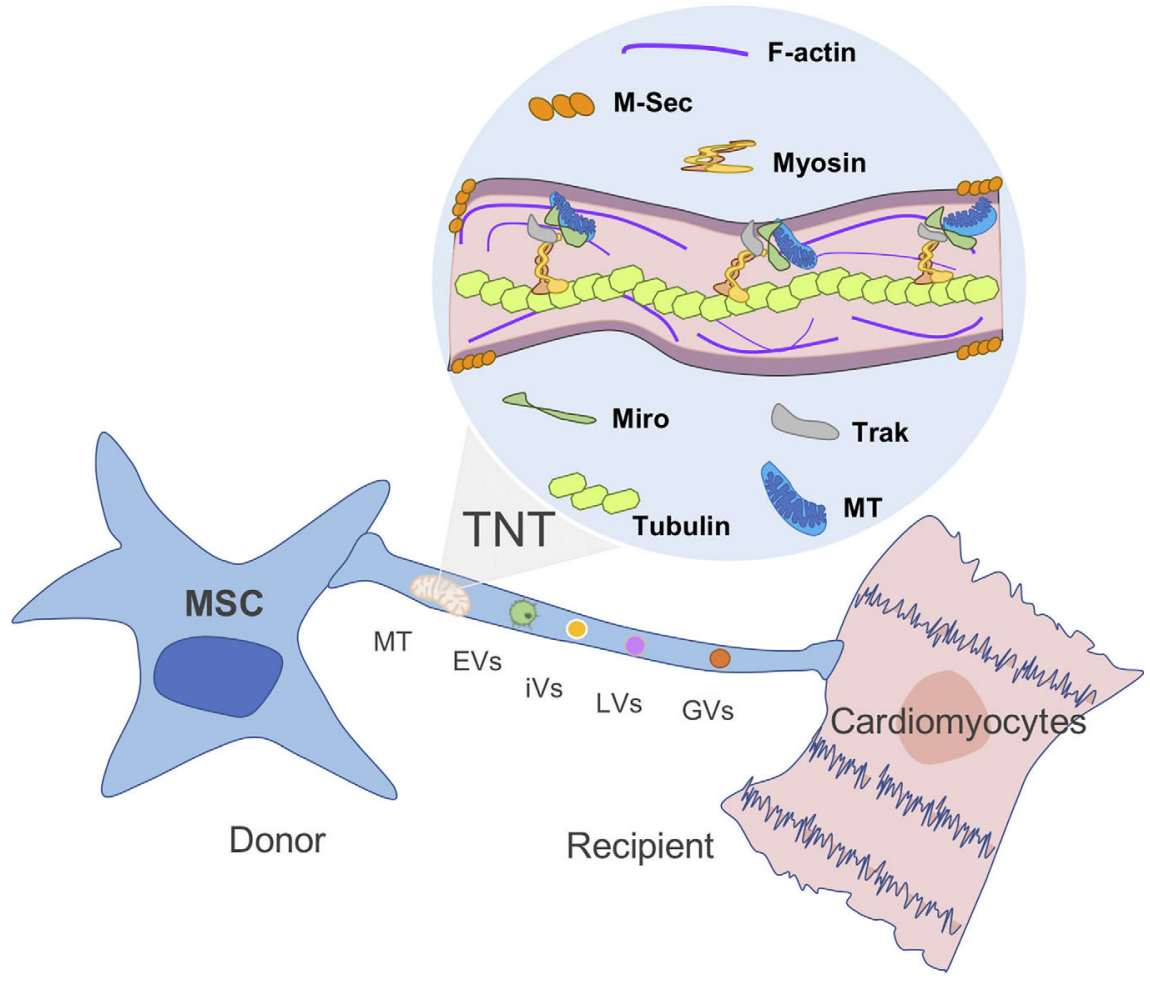

FIGURE 2 | Mitochondria transfer from MSC to recipient cells through TNT. Tunneling nanotubes (TNTS) are small membranous thin cytoplasmic extensions bordered by a plasma membrane and connecting cells of 50-1,000 nm in diameter, containing both F-actin and microtubules. M-sec, a mammalian protein, induces formation of TNTs that only contain actin filaments, but without microtubules. Rho GTPases play an important role in mitochondrial motility through TNT. For instance, Miro1 and microtubules are involved in the regulation of organelle transfer, while Track and Myosin aid to move the mitochondria through the filament (44, 45, 46). MT, mitochondria; EVs, extracellular vesicles; iVs, intracellular vesicles; LVs, lysosomal vesicles; and GVs, Golgi vesicles (GVs). 
directed process and not only a general exchange of cellular contents $(34,45,47-49)$. This method appears to be essential for the transfer of mitochondria in some cell types including MSC, as chemical impairment of the structures reduces significantly mitochondrial exchange (50).

Another mechanism by which MSCs exert their therapeutic effects on recipient cells is via Extracellular Vesicles (EVs) and apoptotic bodies (51). While Phinney et al. studied the effects of MSCs on intracellular stress response, showing that MSCs prompt the movement of depolarized mitochondria into the outer limits of the plasma membrane as a response to a higher concentration of oxygen, in EVs larger than $100 \mathrm{~nm}$ (17). These EVs were loaded with mitochondria and secreted via arrestin domain-containing protein 1 and can fuse with macrophages, enhancing their oxygen consumption rate $(17,52,53)$. The mechanisms of transfer that do not rely on long distance or secreted structures have also been reported in literature. For instance, $\mathrm{Oh}$ et al. has reported cell fusion on a model of myocardial infarction after stem cell therapy between cardiomyocytes and bone marrow transplanted cells (54). On the other hand, gap junctions are a processed by which MSCs attached to cells in regions of high connexin expression, for molecule and organelle exchange (46). Indeed, BMSCs formed Connexin43-mediated gap junctional channels with the alveolar epithelium, which allowed the transfer of mitochondria that led to cellular protection upon infection (46).

\section{ARTIFICIAL TRANSFER TO SELECTIVELY ASSESS THE FUNCTIONAL EFFECT OF MSC-DERIVED MT}

Caicedo et al. used the term of MitoCeption to define the artificial way to transfer MT from a donor into a recipient cell. MSCs derived active mitochondria (MT) were transferred into a MDAMB-231 cancer cell line. The "mitocepted" cells were able to increase glycolytic metabolism as well as their ATP production. Moreover, the recipient MDA cells recovered invasive and proliferative capacities (55). Another way to transfer MT has been described based on a so called "photothermal nanoblade", independent of endocytosis and cell fusion. The nanoblade rescued the pyrimidine auxotroph phenotype and respiration of $\rho 0$ cells that lack mtDNA (56). Of interest, metabolomic analysis of the mitocepted cells showed a change that occurs in the mtDNA haplotype in receptor somatic mammalian cells, suggesting this might be a procedure to treat MT genetic defects. Artificial transfer selectively allows the analysis of the sole impact of MT from MSCs to a specific target cell, without the interference of other parameters such as cell contact and paracrine factors.

\section{FUTURE PERSPECTIVE FOR ENHANCED MSC-DEPENDENT MT TRANSFER}

Different strategies to augment MSC-MT biomass can be considered to reinforce their MT donation potential. This includes their ex vivo pretreatment with specific drugs that can activate AMPK (AMP-activated protein kinase) and the downstream signaling molecules including PGC-1alpha (peroxisome-proliferator-activated receptor gamma co-activatorlalpha) resulting in increasing MT biogenesis $(57,58)$.

Recently, MT were isolated from donor BMSCs and transferred into recipient BMSCs of the same batch and passage (59). The metabolically augmented MSCs through receiving autologous MT exhibited a significantly enhanced proliferation and migration; more importantly, following osteogenic induction, they showed an increased osteogenesis potential through the upregulation of the aerobic metabolism. The transplantation of the modified MSCs into a rat cranial critical-size bone defect model showed an improved bone formation in situ. Increased OXPHOS activity and ATP production were observed, which upon inhibition by oligomycin attenuated all the enhancement functions including the osteogenic differentiation (59).

Mitochondrial diseases are rare genetic disorders that occur when the mitochondria fail to produce enough energy for cell function (60). Mitochondrial diseases can affect almost any tissues, including the neurons (61), myocytes (62), kidney (63). Depending on which cells and tissue type are affected, symptoms may include loss of motor control, muscle weakness and pain, gastrointestinal disorders, intestinal malabsorption syndrome, growth retardation, heart disease, diabetes, visual or hearing impairment, susceptibility to infections, fertility and hormonal disorders, among many other pathologies. Since mitochondria come only from the maternal oocyte these inherited mitochondrial diseases are exclusively matrilineal. Moreover, not all mitochondria are mutated (mitochondrial heteroplasmy), and severity of symptoms depend on the percentage of mutant mitochondria in cells. In these rare disorders, transfer of mitochondria after expansion of donor cells, isolation of organelles and reinfusion through vector or physical cell introduction could be a therapeutical options (64). However, before clinical mitochondrial transfer is applied, many questions still require further investigation, including (a) the therapeutic difference between the use of autologous versus allogeneic MT sources and the effect of the acquired heteroplasmy; (b) the effect of tissue sources and demographic donor variability; (c) the fate and persistence of the donated MT in the recipient cells; and (d) the identification of protein and RNA cargo shuttled by the transferred MT.

\section{CONCLUSIONS}

MSCs, in their natural microenvironment, appear to be primarily glycolytic, but when entering a proliferative state they undergo a metabolic shift toward OXPHOS and increased mitochondrial activity. This OXPHOS metabolic state is associated with increased production of ROS and leads to mitochondria dysfunction. In contrast, MSC adipogenesis or osteoblast differentiation is associated with a decrease of the pentose phosphate pathway (PPP) pathway. In parallel, the 
mitochondrial enzymatic activities are increased with a high oxidative phosphorylation and beta-oxidation.

All the findings detailed in this review are compatible with the notion that MSCs respond to damaged or inflamed tissue through the transfer of MT to injured and immune cells, conveying a type of signaling that contributes to the restoration of cell homeostasis and immune function. The eventual reversal, induced by MT transfer, of the metabolic and pro-inflammatory profile, will open new avenues to the understanding of inflammatory diseases, their relation to both systemic and local risk factors, and also leads to new therapeutic tools for the control of the disease.

\section{REFERENCES}

1. Charbord P, Livne E, Gross G, Häupl T, Neves NM, Marie P, et al. Human Bone Marrow Mesenchymal Stem Cells: A Systematic ReappraisalVia the Genostem Experience. Stem Cell Rev Rep (2011) 7(1):32-42.

2. Zavala G, Sandoval C, Meza D, Contreras R, Gubelin W, Khoury M. Differentiation of adipose-derived stem cells to functional CD105 $5^{\text {neg }}$ $\mathrm{CD}_{73}{ }^{\text {low }}$ melanocyte precursors guided by defined culture condition. Stem Cell Res Ther (2019) 10:249. doi: 10.1186/s13287-019-1364-0

3. González PL, Carvajal C, Cuenca J, Alcayaga-Miranda F, Figueroa FE, Bartolucci J, et al. Chorion mesenchymal stem cells show superior differentiation,immunosuppressive, and angiogenic potentials in comparison with haploidentical maternal placentalcells. Stem Cells Transl Med (2015) 4(10):1109-21. doi: 10.5966/sctm.2015-0022

4. Angelopoulos I, Brizuela C, Khoury M. Gingival Mesenchymal Stem Cells Outperform Haploidentical DentalPulp-derived Mesenchymal Stem Cells in Proliferation Rate, Migration Ability, and AngiogenicPotential. Cell Transplant (2018) 27(6):967-78. doi: 10.1177/0963689718759649

5. Maumus M, Jorgensen C, Noël D. Mesenchymal stem cells in regenerative medicine applied to rheumaticdiseases: Role of secretome and exosomes. Biochimie (2013) 95(12):2229-34. doi: 10.1016/j.biochi.2013.04.017

6. Luz-Crawford P, Torres MJ, Noël D, Fernandez A, Toupet K, AlcayagaMiranda F, et al. The immunosuppressive signature of menstrual blood mesenchymal stemcells entails opposite effects on experimental arthritis and graft versus hostdiseases. Stem Cells (2016) 34(2):456-69. doi: 10.1002/ stem. 2244

7. Buravkova LB, Rylova YV, Andreeva ER, Kulikov AV, Pogodina MV, Zhivotovsky B, et al. Low ATP level is sufficient to maintain the uncommitted state ofmultipotent mesenchymal stem cells. Biochim Biophys Acta - Gen Subj (2013) 17(2):253. doi: 10.1016/j.bbagen.2013.05.029

8. Meleshina AV, Dudenkova VV, Shirmanova MV, Shcheslavskiy VI, Becker W, Bystrova AS, et al. Probing metabolic states of differentiating stem cells usingtwo-photon FLIM. Sci Rep (2016) 6:21853. doi: 10.1038/srep21853

9. Shyh-Chang N, Daley GQ, Cantley LC. Stem cell metabolism in tissue development andaging. Development (2013) 140(12):2535-47. doi: 10.1242/ dev.091777

10. Meleshina AV, Dudenkova VV, Bystrova AS, Kuznetsova DS, Shirmanova MV, Zagaynova EV. Two-photon FLIM of NAD(P)H and FAD in mesenchymal stem cellsundergoing either osteogenic or chondrogenic differentiation. Stem Cell Res Ther (2017) 8:15. doi: 10.1186/s13287-0170484-7

11. Ghannam S, Bouffi C, Djouad F, Jorgensen C, Noël D. Immunosuppression by mesenchymal stem cells: Mechanisms and clinicalapplications. Stem Cell Res Ther (2010) 1(1):2. doi: 10.1186/scrt2

12. Djouad F, Plence P, Bony C, Tropel P, Apparailly F, Sany J, et al. Immunosuppressive effect of mesenchymal stem cells favors tumorgrowth in allogeneic animals. Blood (2003) 02(10):3837-44. doi: 10.1182/blood-200304-1193

13. Contreras-Lopez R, Elizondo-Vega R, Paredes MJ, Luque-Campos N, Torres MJ, Tejedor G, et al. HIF1 $\alpha$-dependent metabolic reprogramming governs mesenchymalstem/stromal cell immunoregulatory functions. FASEB J (2020) 34(6):8250-64. doi: 10.1096/fj.201902232R

\section{AUTHOR CONTRIBUTIONS}

CJ and MK contributed equally to the review. All authors contributed to the article and approved the submitted version.

\section{FUNDING}

This work was supported by grants from National Agency for Investigation and Development: ANID (Agencia Nacional de Investigación y Desarrollo) [FONDECYT regular \#1170852, $\# 1201420$ and \#1211749].

14. Liu Y, Yuan X, Muñoz N, Logan TM, Ma T. Commitment to Aerobic Glycolysis Sustains Immunosuppression of HumanMesenchymal Stem Cells. Stem Cells Transl Med (2019) 8(1):93-106. doi: 10.1002/sctm.18-0070

15. Saxton RA, Sabatini DM. mTOR Signaling in Growth, Metabolism, and Disease.Cell (2017) 168(6):960-76. doi: 10.1016/j.cell.2017.02.004

16. Vigo T, La Rocca C, Faicchia D, Procaccini C, Ruggieri M, Salvetti M, et al. IFN $\beta$ enhances mesenchymal stromal (Stem) cells immunomodulatoryfunction through STAT1-3 activation and mTORassociated promotion of glucosemetabolism. Cell Death Dis (2019) 17 (1):999-1007. doi: 10.1038/s41419-019-1336-4

17. Phinney DG, Di Giuseppe M, Njah J, Sala E, Shiva S, St Croix CM, et al. Mesenchymal stem cells use extracellular vesicles to outsource mitophagy and shuttle microRNAs. Nat Commun (2015) 6:8472. doi: 10.1038/ncomms9472

18. Pattappa G, Heywood HK, de Bruijn JD, Lee DA. The metabolism of human mesenchymal stem cells during proliferationand differentiation. J Cell Physiol (2011) 226(10):25627-70. doi: 10.1002/jcp.22605

19. Ma Y, Qi M, An Y, Zhang L, Yang R, Doro DH, et al. Autophagy controls mesenchymal stem cell properties and senescenceduring bone aging. Aging Cell (2018) 17(1):e12709. doi: 10.1111/acel.12709

20. Chang TC, Hsu MF, Wu KK. High glucose induces bone marrow-derived mesenchymal stem cellsenescence by upregulating autophagy. PloS One (2015) 10(5):e0126537. doi: 10.1371/journal.pone.0126537

21. Yuan X, Logan TM, Ma T. Metabolism in human mesenchymal stromal cells: A missing linkbetween HMSC biomanufacturing and therapy? Front Immunol (2019) 10:977. doi: 10.3389/fimmu.2019.00977

22. Kim YH, Jin HJ, Heo J, Ju H, Lee HY, Kim S, et al. Small hypoxia-primed mesenchymal stem cells attenuate graft-versus-host disease. Leukemia (2018) 32:2672-84. doi: 10.1038/s41375-018-0151-8

23. Ma Z, Song G, Liu D, Qian D, Wang Y, Zhou J, et al. N-Acetylcysteine enhances the therapeutic efficacy of bonemarrow-derived mesenchymal stem cell transplantation in rats with severe acutepancreatitis. Pancreatology (2019) 19(2):258-65. doi: 10.1016/j.pan.2019.01.004

24. Watanabe J, Yamada $M$, Niibe $K$, Zhang $M$, Kondo $T$, Ishibashi $M$, et al. Preconditioning of bone marrow-derived mesenchymal stem cells withNacetyl-L-cysteine enhances bone regeneration via reinforced resistance to oxidativestress. Biomaterials (2018) 1185:25-38. doi: 10.1016/ j.biomaterials.2018.08.055

25. Kim DS, Jang IK, Lee MW, Ko YJ, Lee DH, Lee JW, et al. Enhanced Immunosuppressive Properties of Human Mesenchymal StemCells Primed by Interferon- $\gamma$. EBioMedicine (2018) 28:261-73. doi: 10.1016/ j.ebiom.2018.01.002

26. Luz-Crawford P, Ipseiz N, Espinosa-Carrasco G, Caicedo A, Tejedor G, Toupet $K$, et al. PPAR $\beta / \delta$ directs the therapeutic potential of mesenchymalstem cells in arthritis. Ann Rheum Dis (2016) 75(12):2166-74. doi: 10.1136/annrheumdis-2015-208696

27. Lee SY, Long F. Notch signaling suppresses glucose metabolism in mesenchymalprogenitors to restrict osteoblast differentiation. J Clin Invest (2018) 128(12):5573-86. doi: 10.1172/JCI96221

28. Zheng J, Li H, He L, Huang Y, Cai J, Chen L, et al. Preconditioning of umbilical cord-derived mesenchymal stem cells byrapamycin increases cell migration and ameliorates liver ischaemia/reperfusion injury in mice via the CXCR4/CXCL12 axis. Cell Prolif (2019) 52(2):e12546. doi: 10.1111/cpr.12546 
29. Caicedo A, Aponte PM, Cabrera F, Hidalgo C, Khoury M. Artificial Mitochondria Transfer: Current Challenges, Advances, andFuture Applications. Stem Cells Int (2017) 2017:7610414. doi: 10.1155/2017/7610414

30. Naimul Islam M, Otsu K, Houser SD, Lindert J, Bhattacharya J. Mitochondrial donation by mesenchymal stromal cells rescues alveolarsurfactant secretion in sepsis. FASEB J (2010) 12.24-612.24. doi: 10.1096/fasebj.24.1_supplement.612.24

31. Khoury M, Cuenca J, Cruz FF, Figueroa FE, Rocco PRM, Weiss DJ. Current Status of Cell-Based Therapies for Respiratory VirusInfections: Applicability to COVID19. Eur Respir J (2020) 55(6):2000858. doi: 10.1183/13993003.00858-2020

32. Cabrera F, Ortega M, Velarde F, Parra E, Gallardo S, Barba D, et al. Primary allogeneic mitochondrial mix (PAMM) transfer/transplant by MitoCeption to address damage in PBMCs caused by ultraviolet radiation. BMC Biotechnol (2019) 19(1):42. doi: 10.1186/s12896-019-0534-6

33. Islam MN, Das SR, Emin MT, Wei M, Sun L, Westphalen K, et al. Mitochondrial transfer from bone-marrow-derived stromal cells topulmonary alveoli protects against acute lung injury. Nat Med (2012) 8 (5):759-65. doi: 10.1038/nm.2736

34. Ahmad T, Mukherjee S, Pattnaik B, Kumar M, Singh S, Rehman R, et al. Miro1 regulates intercellular mitochondrial transport \& enhances mesenchymal stem cell rescue efficacy. EMBO J (2014) 33(9):994-1010. doi: $10.1002 / \mathrm{embj} .201386030$

35. Vallabhaneni KC, Haller H, Dumler I. Vascular smooth muscle cells initiate proliferation of mesenchymal stem cells by mitochondrial transfer via tunneling nanotubes. Stem Cells Dev (2012) 21(17):3104-13. doi: 10.1089/ scd.2011.0691

36. Zheng Y, Cantley LC. Toward a better understanding of folate metabolism in health anddisease. J Exp Med (2019) 216(2):253-66. doi: 10.1084/jem.20181965

37. Jackson MV, Morrison TJ, Doherty DF, McAuley DF, Matthay MA, Kissenpfennig A, et al. Mitochondrial Transfer via Tunneling Nanotubes is an ImportantMechanism by Which Mesenchymal Stem Cells Enhance Macrophage Phagocytosis in the In Vitro and InVivo Models of ARDS. Stem Cells (2016) 34(8):2210-23. doi: 10.1002/stem.2372

38. Luz-Crawford P, Noël D, Fernandez X, Khoury M, Figueroa F, Carrión F, et al. Mesenchymal Stem Cells Repress Th17 Molecular Program through the PD-1 Pathway. PloS One (2012) 7(9):e45272. doi: 10.1371/journal.pone.0045272

39. Polchert D, Sobinsky J, Douglas G, Kidd M, Moadsiri A, Reina E, et al. IFNgamma activation of mesenchymal stem cells for treatment and prevention of graft versus host disease. Eur J Immunol (2008) 38:1745-55. doi: 10.1002/ eji.200738129

40. Luz-Crawford P, Hernandez J, Djouad F, Luque-Campos N, Caicedo A, Carrère-Kremer S, et al. Mesenchymal stem cell repression of Th17 cells is triggered bymitochondrial transfer. Stem Cell Res Ther (2019) 10(1):232. doi: 10.1186/s13287-019-1307-9

41. Court AC, Le-Gatt A, Luz-Crawford P, Parra E, Aliaga-Tobar V, Bátiz LF, et al. Mitochondrial transfer from MSCs to T cells induces Tregdifferentiation and restricts inflammatory response. EMBO Rep (2020) 21(2):e48052. doi: 10.15252/embr.201948052

42. Mahrouf-Yorgov M, Augeul L, Da Silva CC, Jourdan M, Rigolet M, Manin S, et al. Mesenchymal stem cells sense mitochondria released from damagedcells as danger signals to activate their rescue properties. Cell Death Differ (2017) 24 (7):1224-38. doi: 10.1038/cdd.2017.51

43. Jackson M, Krasnodembskaya A. Analysis of Mitochondrial Transfer in Direct Co-cultures of HumanMonocyte-derived Macrophages (MDM) and Mesenchymal Stem Cells (MSC). Bio Protoc (2017) 7(9):e2255. doi: 10.21769/bioprotoc.2255

44. Rustom A, Saffrich R, Markovic I, Walther P, Gerdes HH. Nanotubular Highways for Intercellular OrganelleTransport. Science 80- (2004) 303 (5660):1007-10. doi: 10.1126/science.1093133

45. Hase K, Kimura S, Takatsu H, Ohmae M, Kawano S, Kitamura H, et al. M-Sec promotes membrane nanotube formation by interacting with Ral and the exocyst complex. Nat Cell Biol (2009) 11:1427-32. doi: 10.1038/ncb1990

46. Sinclair KA, Yerkovich ST, Hopkins PMA, Chambers DC. Characterization of intercellular communication and mitochondrialdonation by mesenchymal stromal cells derived from the human lung. Stem Cell Res Ther (2016) 7:91. doi: 10.1186/s13287-016-0354-8

47. Quintero OA, DiVito MM, Adikes RC, Kortan MB, Case LB, Lier AJ, et al. Human Myo19 Is a Novel Myosin that Associates with Mitochondria. Curr Biol (2009) 19:2008-13. doi: 10.1016/j.cub.2009.10.026
48. Brickley K, Stephenson FA. Trafficking kinesin protein (TRAK)-mediated transport of mitochondria in axons of hippocampal neurons. J Biol Chem (2011) 286:18079-92. doi: 10.1074/jbc.M111.236018

49. Chang KT, Niescier RF, Min K-T. Mitochondrial matrix Ca2+ as an intrinsic signal regulating mitochondrial motility in axons. Proc Natl Acad Sci (2011) 108:15456-61. doi: 10.1073/pnas.1106862108

50. Domhan S, Ma L, Tai A, Anaya Z, Beheshti A, Zeier M, et al. Intercellular communication by exchange of cytoplasmic material viatunneling nano-tube like structures in primary human renal epithelial cells. PloS One (2011) 6(6): e21283. doi: 10.1371/journal.pone.0021283

51. Morrison TJ, Jackson MV, Cunningham EK, Kissenpfennig A, McAuley DF, O'Kane CM, et al. Mesenchymal stromal cells modulate macrophages in clinicallyrelevant lung injury models by extracellular vesicle mitochondrial transfer. Am J Respir Crit Care Med (2017) 196(10):1275-86. doi: 10.1164/ rccm.201701-0170OC

52. Torralba D, Baixauli F, Sánchez-Madrid F. Mitochondria Know No Boundaries: Mechanisms and Functions of Intercellular Mitochondrial Transfer. Front Cell Dev Biol (2016) 4:107:107. doi: 10.3389/fcell.2016.00107

53. Wang Y, Branicky R, Noë A, Hekimi S. Superoxide dismutases: Dual roles in controlling ROS damage and regulating ROS signaling. J Cell Biol (2018) 217:1915-28. doi: 10.1083/jcb.201708007

54. Oh H, Bradfute SB, Gallardo TD, Nakamura T, Gaussin V, Mishina Y, et al. Cardiac progenitor cells from adult myocardium: homing, differentiation, and fusion after infarction. Proc Natl Acad Sci U S A (2003) 100:12313-8. doi: $10.1073 /$ pnas. 2132126100

55. Caicedo A, Fritz V, Brondello J-M, Ayala M, Dennemont I, Abdellaoui N, et al. MitoCeption as a new tool to assess the effects of mesenchymal stem/ stromal cell mitochondria on cancer cell metabolism and function. Sci Rep (2015) 5:9073. doi: 10.1038/srep09073

56. Wu TH, Sagullo E, Case D, Zheng X, Li Y, Hong JS, et al. Mitochondrial Transfer by Photothermal Nanoblade Restores MetaboliteProfile in Mammalian Cells. Cell Metab (2016) 23(5):921-9. doi: 10.1016/j.cmet.2016.04.007

57. Chen CT, Hsu SH, Wei YH. Mitochondrial bioenergetic function and metabolic plasticity in stemcell differentiation and cellular reprogramming. Biochim Biophys Acta - GenSubj (2012) 1820(5):571-6. doi: 10.1016/j.bbagen.2011.09.013

58. Jornayvaz FR, Shulman GI. Regulation of mitochondrial biogenesis.Essays Biochem (2010) 47:69-84. doi: 10.1042/BSE0470069

59. Guo Y, Chi X, Wang Y, Heng BC, Wei Y, Zhang X, et al. Mitochondria transfer enhances proliferation, migration, and osteogenic differentiation of bone marrow mesenchymal stem cell and promotes bone defect healing. Stem Cell Res Ther (2020) 11. doi: 10.1186/s13287-020-01704-9

60. Schapira AHV. Mitochondrial diseases. Lancet (2012) 79(9828):1825-34. doi: 10.1016/S0140-67361161305-6

61. Johri A, Beal MF. Mitochondrial dysfunction in neurodegenerativediseases. J Pharmacol Exp Ther (2012) 342(3):619-30. doi: 10.1124/jpet.112.192138

62. Bai R, Higgs JD. Mitochondrial disorders. In: . MolecularPathology in Clinical Practice:Second Edition (2016) 139-59. doi: 10.1007/978-3-319-19674-9_10

63. Emma F, Montini G, Parikh SM, Salviati L. Mitochondrial dysfunction in inherited renal disease and acute kidney injury. Nat Rev Nephrol (2016). doi: $10.1038 /$ nrneph.2015.214

64. Lin TK, Chen SD, Chuang YC, Lan MY, Chuang JH, Wang PW, et al. Mitochondrial transfer of wharton's jelly mesenchymal stem cells eliminates mutation burden and rescues mitochondrial bioenergetics in rotenone-stressed MELAS fibroblasts. Oxid Med Cell Longev (2019). doi: 10.1155/2019/9537504

Conflict of Interest: MK is the chief scientific officer of Cells for Cells and Regenero, the chilean consortium for regenerative medicine.

The remaining authors declare that the research was conducted in the absence of any commercial or financial relationships that could be construed as a potential conflict of interest.

Copyright (c) 2021 Jorgensen and Khoury. This is an open-access article distributed under the terms of the Creative Commons Attribution License (CC BY). The use, distribution or reproduction in other forums is permitted, provided the original author(s) and the copyright owner(s) are credited and that the original publication in this journal is cited, in accordance with accepted academic practice. No use, distribution or reproduction is permitted which does not comply with these terms. 